\title{
Bounded Continuously Distributed Delays in Dynamic Oligopolies
}

\author{
By \\ Carl Chiarella ${ }^{1 *}$ and Ferenc Szidarovszky\# \\ *School of Finance and Economics, University of Technology, Sydney \\ P.O. Box 123, Broadway, NSW 2007, AUSTRALIA \\ E-mail: carl.chiarella@uts.edu.au \\ \#Systems and Industrial Engineering Department, University of Arizona \\ Tucson, Arizona 85721-0020, U.S.A. \\ E-mail: szidar@sie.arizona.edu
}

\begin{abstract}
This paper introduces a way of modelling bounded continuously distributed time lags in dynamic economic models. Past data are averaged only over a bounded interval thus avoiding the use of very old ("stale") economic data and hence making dynamic economic models more realistic. Dynamic oligopolies are formulated and then examined under this new type of information lag. Stability analysis is presented and the possibility of the birth of limit cycles is examined. Some special cases are considered, the computational analysis of which illustrates the theoretical findings.

Acknowledgements. The authors are indebted to Dr. Peiyuan Zhu for writing the computer programs that generated the diagrams. The authors also with to thank Professor Laura Gardini for a number of very insightful comments that helped to clarify some misconceptions in an earlier version of this paper.
\end{abstract}

\footnotetext{
${ }^{1}$ Corresponding author: E-mail: carl.chiarella@uts.edu.au
} 


\section{Introduction}

Dynamic oligopoly models have been examined by many researchers during the last three decades. The most important references and results on single-product oligopolies are summarised in Okuguchi (1976) and their extensions to the multi-product case are discussed in Okuguchi and Szidarovszky (1999). Most of the earlier models were based on the special assumption that at each time period all relevant information is instantaneously available. However in economic reality there is always a time lag in obtaining and implementing information about the rivals' output as well as about the firms' own output. Time delays can be modelled either by assuming fixed time lags or continuously distributed time lags. Russel et al. (1986) used fixed time lags and their dynamic model was described by a differential-difference equation. The major difficulty in using this idea arises from the fact that time delays are not known exactly. Continuously distributed time lags are more realistic. They have been earlier introduced and used in mathematical biology (see for example, Cushing, 1977), and their first economic application was presented by Invernizzi and Medio (1991). Based on their ideas Chiarella and Khomin (1996) and Chiarella and Szidarovszky (2001) have examined dynamic oligopolies with continuously distributed time lags and examined the asymptotic behaviour of the equilibrium. Continuously distributed time lags are based on the weighted average of all past data from time zero up to the current time period $t$. For larger values of $t$ this assumption seems unrealistic since very early, irrelevant data are also used in calculating the current average. In addition, dynamic economic models with continuously distributed time lags right back to the beginning of the process generally tend to be highly stable. The aim of this paper is to introduce continuously distributed lags over a finite time interval. Dynamic oligopoly models will be formulated with these bounded continuously distributed lags and their stability properties, particularly in relation to the possible birth of limit cycles, will be studied and compared with the infinitely continuously distributed lag case considered by Chiarella and Szidarovszky (2001). 
This paper develops as follows. In section 2 bounded continuously distributed lags will be introduced, and in section 3 will be applied to the corresponding dynamic oligopoly models. We will also show that the resulting integro-differential equations are equivalent to a certain system of ordinary differential-difference equations. In section 4 stability analysis on the basis of local linearization will be undertaken, and the Hopf bifurcation theorem will be applied in section 5 to examine the possibility of the birth of limit cycles. Some important special cases will be discussed in section 6 , and in sections 7 and 8 computational results will illustrate the theoretical findings. Section 9 will conclude the paper.

\section{Bounded Continuously Distributed Lags}

Select a positive constant $\delta>0$, and assume that the delayed information on any function $x(t)$ is given by a weighted average of the past data $x(s)$ for $s \in[t-\delta, t]$. The weighting function of infinitely continuously distributed lags used in Invernizzi and Medio (1991), Chiarella and Khomin (1996) and Chiarella and Szidarovszky (2001) cannot be used here, since its integral equals $l$ on the interval $[0, \infty]$, rather than on the new interval $[t-\delta$, $t$ ]. However we may still use basically the same weighting function if an appropriate normalising factor is introduced. Hence in this paper we will use weighting functions of the form

$$
w(t-s, T, 0, \delta)=\frac{1}{C(0, T, \delta)} \cdot \frac{1}{T} e^{-\frac{(t-s)}{T}} \text { for } m=0
$$

and

$$
w(t-s, T, m, \delta)=\frac{1}{C(m, T, \delta)} \cdot \frac{1}{m !}\left(\frac{m}{T}\right)^{m+1}(t-s)^{m} e^{-\frac{m(t-s)}{T}} \quad \text { for } m=1,2, \ldots
$$

Here $C(m, T, \delta)$ is the normalising factor and we note that functions (1) and (2) are the $1 / C(m, T, \delta)$-multiples of the corresponding weighting functions used by Invernizzi and Medio (1991), Chiarella and Khomin (1996) and Chiarella and Szidarovszky (2001) in 
modelling continuously distributed time lags. The weighting functions have the following properties:

i. for $m=0$, weights are exponentially declining with the most weight given to the most current data;

ii. for $m \geq 1$, zero weight is assigned to the most recent output, rising to maximum at $t-s$ $=T$, and declining exponentially thereafter;

iii. as $m$ increases, the function becomes more peaked around $t-s=T$. For sufficiently large values of $m$ the function may for all practical purposes be regarded as very close to the Dirac delta function centered at $t-S=T$;

iv. as $T \rightarrow 0$, the function tends to the Dirac delta function.

First we establish the functional form of the normalising factors.

\section{Lemma 1.}

The normalising factors are given by

$$
C(0, T, \delta)=1-e^{-\frac{\delta}{T}} \text { for } m=0,
$$

and for $m \geq 1$, by

$$
C(m, T, \delta)=1-e^{-\frac{m \delta}{T}} \sum_{k=0}^{m} \frac{\left(\frac{m \delta}{T}\right)^{k}}{k !} .
$$

\section{Proof.}

The normalising factors result from the requirement that $\int_{t-\delta}^{t} w(t-s, T, m) d s=1$ for all $m$.

Notice first that

$$
C(0, T, \delta)=\int_{t-\delta}^{t} \frac{1}{T} e^{-\frac{t-s}{T}} d s=\int_{\delta}^{0}-\frac{1}{T} e^{-\frac{u}{\delta}} d u=1-e^{-\frac{\delta}{T}},
$$

when we introduce the new variable $u=t-s$.

In the general case notice first that

$$
\begin{aligned}
C(m, T, \delta) & =\int_{t-\delta}^{t} \frac{1}{m !}\left(\frac{m}{T}\right)^{m+1}(t-s)^{m} e^{-\frac{m(t-s)}{T}} d s \\
& =\int_{0}^{\delta} \frac{1}{m !}\left(\frac{m}{T}\right)^{m+1} u^{m} e^{-\frac{m u}{T}} d u
\end{aligned}
$$


and by introducing the integrals

$$
C_{k}=\int_{0}^{\delta} \frac{1}{k !}\left(\frac{m}{T}\right)^{k+1} u^{k} e^{-\frac{m u}{T}} d u \quad(k=0,1,2, \ldots, m)
$$

we see that $C(m, T, \delta)=C_{m}$.

Simple integration shows that

$$
C_{0}=\int_{0}^{\delta} \frac{m}{T} e^{-\frac{m u}{T}} d u=1-e^{-\frac{m \delta}{T}}
$$

and by using integration by parts we have for all $k \geq 1$,

$$
\begin{aligned}
C_{k} & =\left[\frac{1}{k !}\left(\frac{m}{T}\right)^{k+1} u^{k} \frac{e^{-\frac{m u}{T}}}{-\frac{m}{T}}\right]_{0}^{\delta}-\int_{0}^{\delta} \frac{1}{k !}\left(\frac{m}{T}\right)^{k+1} k u^{k-1} \frac{e^{-\frac{m u}{T}}}{-\frac{m}{T}} d u \\
& =-\frac{1}{k !}\left(\frac{m}{T}\right)^{k} \delta^{k} e^{-\frac{m \delta}{T}}+C_{k-1} .
\end{aligned}
$$

The assertion follows by adding this equality for $k=1,2, \ldots, m$.

We illustrate the bounded continuously distributed lag weighting function $w(t-s, T, m)$ in Figure 1 for $m$ varying from 0 to 10 at various values of $\delta$. We note that the maximum (for $m>0)$ of $w(t-s, T, m)$ occurs at $T$ and here we have taken $T=1^{2}$. So for values of $\delta<1$, the past values are given an increasing weight as one moves from $t$ to $t$ - $\delta$; this is merely a reflection of the fact that over such a (relatively) short interval the most recent observations may be regarded as less reliable or less certain by the economic agents. As $\delta$ increases past $T$, the weighting function then drops off. In this case agents give less weight to most recent information for reasons previously stated; they give maximum weight to information around lag $T$; information beyond lag $T$ is given decreasing weight being regarded as less relevant to current circumstances. Thus Figure 1 illustrates clearly how the bounded continuously distributed lag weighting function allows economic agents to concentrate on those parts of past information that are considered most relevant and weight that information as they feel appropriate by adjusting the parameter $m$.

\footnotetext{
${ }^{2}$ Since the time scale can always be adjusted to make $T=1$, Figures 1 and 2 can be regarded as giving a feel for the general nature of the family of weighting functions.
} 
It is worth pointing out that for all $m \geq 0$

$$
\lim _{\delta \rightarrow \infty} C(m, T, \delta)=1
$$

so that in this limit the weighting functions (1) and (2) reduce to the infinitely continuously distributed lag case.

In Figure 2 we compare the cases $\delta=5$ and $\delta=\infty$, still for $T=1$. There is essentially no difference between the two weighting functions. This observation will be useful later when we seek to illustrate the difference in dynamic behaviour between the bounded and infinitely continuously distributed lag situations.
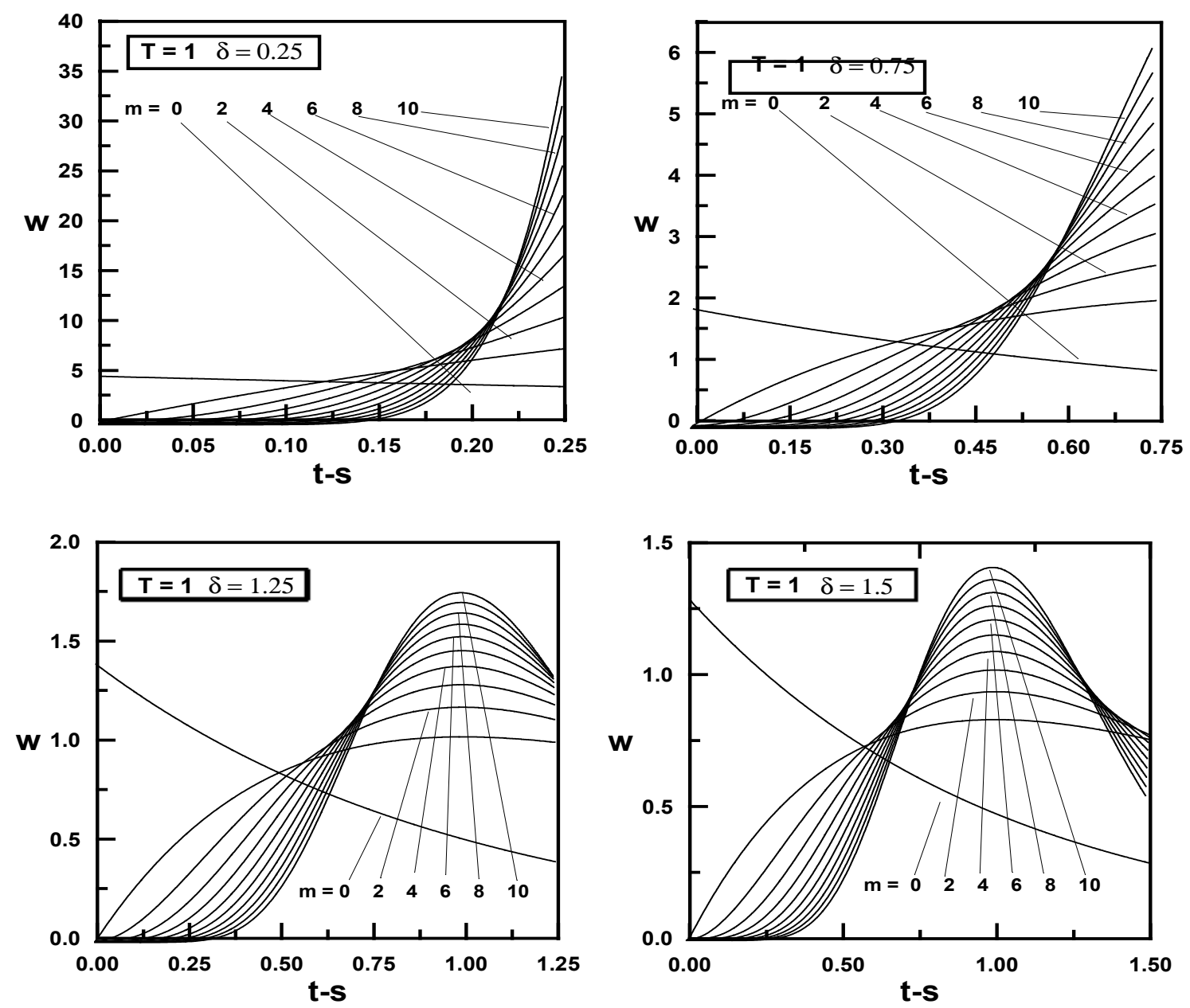

Figure 1. The Bounded Continuously Distributed Lag Weighting Function for Various values of $\delta$ and $m$. 

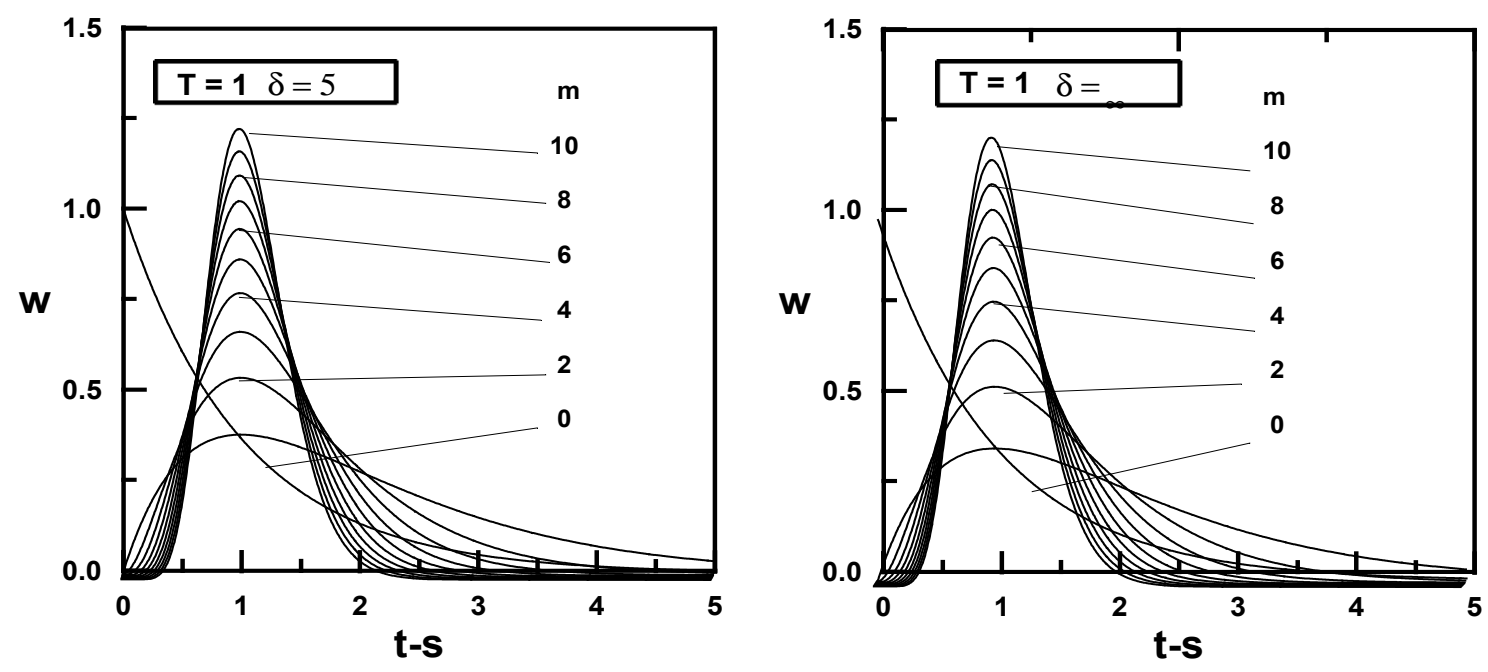

Figure 2. Comparing Large $\delta$ with the Infinitely Continuously Distributed Lag Weighting Function

\section{Dynamic Oligopolies}

Consider an $n$-firm single-product oligopoly without product differentiation in which $x_{i}(t)$ is the output of firm $i$ at time period $t, Q_{i}(t)=\sum_{j \neq i} x_{j}(t)$ is the output of the rest of the industry, and $g_{i}\left(Q_{i}(t)\right)$ is the best response of firm $i$. The equilibrium of the oligopoly is a constant vector $\underline{x}^{*}=\left(x_{1}^{*}, \ldots, x_{n}^{*}\right)$ such that for all $i=1,2, \ldots, n$,

$$
x_{i}^{*}=g_{i}\left(\sum_{j \neq i} x_{j}^{*}\right) .
$$

In the local stability analysis to be considered in subsequent sections, the slopes of the best response functions at the equilibrium, namely

$$
\gamma_{i}=g_{i}^{\prime}\left(Q_{i}^{*}\right)
$$

play an important role. In this paper we only consider oligopolies for which the best response function is downward sloping, so $\gamma_{i}<0$.

Assume that at each time period each firm adjusts its output in the direction of the expected best response then the following dynamic equations are obtained:

$$
\dot{x}_{i}(t)=k_{i}\left(g_{i}\left(Q_{i}^{e}(t)\right)-x_{i}^{e}(t)\right) \quad(i=1,2, \ldots, n)
$$


where $k_{i}>0$ is a given speed of adjustment for each firm. Here $Q_{i}^{e}(t)$ and $x_{i}^{e}(t)$ are respectively firm $i$ 's expectation of rest of industry output and its own output at time t. Both $Q_{i}^{e}(t)$ and $x_{i}^{e}(t)$ are based on delayed information, and the delays are bounded continuously distributed as described in Section 2. Thus

$$
Q_{i}^{e}(t)=\int_{t-\delta_{i}}^{t} w\left(t-s, T_{i}, m_{i}, \delta_{i}\right) Q_{i}(s) d s
$$

and

$$
x_{i}^{e}(t)=\int_{t-\Delta_{i}}^{t} w\left(t-s, S_{i}, l_{i}, \Delta_{i}\right) x_{i}(s) d s .
$$

We use $\delta_{i}$ and $\Delta_{i}$ to respectively denote for firm $i$ the length of the interval over which past rest of industry output and its own output enter its decision process. As Figure 1 indicates, the values of $T_{i}$ and $S_{i}$ show that among all past data being considered, $Q_{i}\left(t-T_{i}\right)$ and $x_{i}\left(t-S_{i}\right)$ have the largest weights for $m_{i} \geq 1$ and $l_{i} \geq 1$. The values of $m_{i}$ and $l_{i}$ indicate how peaked the weighting functions are around $T_{i}$ and $S_{i}$, respectively.

Substituting these equations into (3) a non-linear integro-differential equation system is obtained, the local stability of which is the subject of the next section.

It is clear that a constant vector $\underline{x}^{*}=\left(x_{1}^{*}, \ldots, x_{n}^{*}\right)$ is an equilibrium of the $n$-firm oligopoly if and only if it is the steady-state of the dynamic system (3).

We will next show that all techniques for analysing the asymptotic properties of ordinary differential-difference equations (expounded by Bellman and Cooke, 1963) can be applied by verifying that equations (3) are equivalent to a system of non-linear ordinary differential-difference equations.

Lemma 2. The system of integro-differential equations (3) is equivalent to the system of $3 n+\sum_{i=1}^{n}\left(m_{i}+l_{i}\right)$ ordinary differential-difference equations (8), (9), (15), (16) and (20) below. 


\section{Proof:}

Assume first that $m_{i} \geq 1$. For $k=1,2, \ldots, m_{i}$ introduce functions

$$
q_{i, m_{i}}^{(k)}(t)=\int_{t-\delta_{i}}^{t} \frac{1}{k !}\left(\frac{m_{i}}{T_{i}}\right)^{k+1}(t-s)^{k} e^{-\frac{m_{i}(t-s)}{T_{i}}} Q_{i}(s) d s,
$$

then the definition of the weighting functions $w$ implies that

$$
Q_{i}^{e}(t)=\frac{1}{C\left(m_{i}, T_{i}, \delta_{i}\right)} q_{i, m_{i}}^{\left(m_{i}\right)}(t) .
$$

By simple differentiation we see that

$$
\dot{q}_{i, m_{i}}{ }^{(0)}(t)=\frac{m_{i}}{T_{i}}\left[\sum_{j \neq i} x_{j}(t)-q_{i, m_{i}}^{(0)}(t)\right]-\frac{m_{i}}{T_{i}} e^{-\frac{m_{i} \delta_{i}}{T_{i}}} \sum_{j \neq i} x_{j}\left(t-\delta_{i}\right)
$$

and for $k \geq 1$

$$
\dot{q}_{i, m_{i}}{ }^{(k)}(t)=\frac{m_{i}}{T_{i}}\left[q_{i, m_{i}}^{(k-1)}(t)-q_{i, m_{i}}^{(k)}(t)\right]-\frac{1}{k !}\left(\frac{m_{i}}{T_{i}}\right)^{k+1} \delta_{i}^{k} e^{-\frac{m_{i} \delta_{i}}{T_{i}}} \sum_{j \neq i} x_{j}\left(t-\delta_{i}\right) .
$$

In the case of $m_{i}=0$, we have

$$
Q_{i}^{e}(t)=\frac{1}{C\left(0, T_{i}, \delta_{i}\right)} q_{i, 0}^{(0)}(t)
$$

with

$$
q_{i, 0}^{(0)}(t)=\int_{t-\delta_{i}}^{t} \frac{1}{T_{i}} e^{-\frac{(t-s)}{T_{i}}} Q_{i}(s) d s .
$$

Then simple differentiation shows that

$$
\dot{q}_{i, 0}^{(0)}(t)=\frac{1}{T_{i}}\left[\sum_{j \neq i} x_{j}(t)-q_{i, 0}^{(0)}(t)\right]-\frac{1}{T_{i}} e^{-\frac{\delta_{i}}{T_{i}}} \sum_{j \neq i} x_{j}\left(t-\delta_{i}\right) .
$$

Assume now that $l_{i} \geq 1$. Similarly to the previous derivation introduce functions

$$
x_{i, l_{i}}^{(k)}(t)=\int_{t-\Delta_{i}}^{t} \frac{1}{k !}\left(\frac{l_{i}}{S_{i}}\right)^{k+1}(t-s)^{k} e^{-\frac{l_{i}(t-s)}{S_{i}}} x_{i}(s) d s,
$$

then obviously

$$
x_{i}^{e}(t)=\frac{1}{C\left(l_{i}, S_{i}, \Delta_{i}\right)} x_{i, l_{i}}{ }^{\left(l_{i}\right)}(t)
$$


and similar equations hold for $\dot{x}_{i, l_{i}}^{(k)}(t)$. Thus

$$
\dot{x}_{i, l_{i}}^{(0)}(t)=\frac{l_{i}}{S_{i}}\left[x_{i}(t)-x_{i, l_{i}}^{(0)}(t)\right]-\frac{l_{i}}{S_{i}} e^{-\frac{l_{i} \Delta_{i}}{S_{i}}} x_{i}\left(t-\Delta_{i}\right)
$$

and for $k \geq 1$,

$$
\dot{x}_{i, l_{i}}^{(k)}(t)=\frac{l_{i}}{S_{i}}\left[x_{i, l_{i}}^{(k-1)}(t)-x_{i, l_{i}}^{(k)}(t)\right]-\frac{1}{k !}\left(\frac{l_{i}}{S_{i}}\right)^{k+1} \Delta_{i}^{k} e^{-\frac{l_{i} \Delta_{i}}{S_{i}}} x_{i}\left(t-\Delta_{i}\right) .
$$

In the case of $l_{i}=0$, we have

$$
x_{i}^{e}(t)=\frac{1}{C\left(0, S_{i}, \Delta_{i}\right)} x_{i, 0}^{(0)}(t)
$$

with

$$
x_{i, 0}^{(0)}(t)=\int_{t-\Delta_{i}}^{t} \frac{1}{S_{i}} e^{-\frac{(t-s)}{S_{i}}} x_{i}(s) d s .
$$

By simple differentiation

$$
\dot{x}_{i, 0}^{(0)}(t)=\frac{1}{S_{i}}\left[x_{i}(t)-x_{i, 0}^{(0)}(t)\right]-\frac{1}{S_{i}} e^{-\frac{\Delta_{i}}{S_{i}}} x_{i}\left(t-\Delta_{i}\right) .
$$

By using relations (7) and (14) we can rewrite the dynamic equation (3) in terms of the new variables as

$$
\dot{x}_{i}(t)=k_{i}\left[g_{i}\left(\frac{1}{C\left(m_{i}, T_{i}, \delta_{i}\right)} q_{i, m_{i}}^{\left(m_{i}\right)}(t)\right)-\frac{1}{C\left(l_{i}, S_{i}, \Delta_{i}\right)} x_{i, l_{i}}^{\left(l_{i}\right)}(t)\right] .
$$

Then equations (8), (9), (15), (16) and (20) give a system of ordinary differentialdifference equations ${ }^{3}$ for the unknown functions $q_{i, m_{i}}^{(k)}(t) \quad\left(k=0,1, \ldots, m_{i}\right)$, $x_{i, l_{i}}^{(k)}(t) \quad\left(k=0,2, \ldots, l_{i}\right)$, and $x_{i}(t)$ for $i=1,2, \ldots, n$ with $Q_{i}^{e}(t)$ and $x_{i}^{e}(t)$ calculated from (7) and (14) respectively. Notice that the number of equations equals the number of unknowns, namely

$$
\sum_{i=1}^{n}\left(\left(m_{i}+1\right)+\left(l_{i}+1\right)+1\right)=3 n+\sum_{i=1}^{n}\left(m_{i}+l_{i}\right)
$$

\footnotetext{
${ }^{3}$ The special subcases when $m_{i}=0$ and/or $l_{i}=0$ are obtained by using (10)-(12) and (17)-(19) as appropriate.
} 
The initial conditions are selected as follows:

$$
\begin{aligned}
& q_{i}^{(k)}(0)=0 \quad(\text { for all } k) \\
& x_{i}^{(k)}(0)=0 \quad(\text { for all } k) \\
& x_{i}(\tau)=\text { close to equilibrium }(-\varepsilon \leq \tau \leq 0)
\end{aligned}
$$

where $\varepsilon=\max \left\{\delta_{1}, \ldots, \delta_{n}, \Delta_{1}, \ldots, \Delta_{n}\right\}$.

\section{Local Stability Analysis}

As we have demonstrated in the previous section the integro-differential equation system (3) is equivalent to a system of ordinary differential-difference equations, therefore the same methods can now be applied as used for the stability analysis of ordinary differential-difference equations.

Using $x_{i}(t)$ and $Q_{i}(t)$ now to denote deviations of these variables from their equilibrium levels, the linearization of equations (3) reads

$$
\begin{aligned}
\dot{x}_{i}(t)=k_{i}\left\{\gamma_{i} \int_{t-\delta_{i}}^{t} w\left(t-s, T_{i}, m_{i}, \delta_{i}\right) Q_{i}(s) d s\right. \\
\left.-\int_{t-\Delta_{i}}^{t} w\left(t-\Delta, S_{i}, l_{i}, \Delta_{i}\right) x_{i}(s) d s\right\},
\end{aligned}
$$

where $w(t-s, T, m)$ is as defined previously, furthermore $\gamma_{i}$ is the derivative of the best response $g_{i}$ of firm $i$ at the equilibrium. We now state the main result on the eigenvalue structure of the system (21):

Theorem 1. The eigenvalue spectrum of the linearized equation (21) is given by the solutions of the polynomial-exponential equation

$$
\prod_{j=1}^{n}\left(A_{j}(\lambda)-B_{j}(\lambda)\right)\left[1+\sum_{j=1}^{n} \frac{B_{j}(\lambda)}{A_{j}(\lambda)-B_{j}(\lambda)}\right]=0
$$

where the quantities $A_{i}(\lambda), B_{i}(\lambda)$ are defined by equations (25), (26) below.

\section{$\underline{\text { Proof }}$}

We look for the solution in the form 


$$
x_{i}(t)=v_{i} e^{\lambda t} \quad(i=1,2, \ldots, n)
$$

as in Bellman and Cooke (1963), then equation (21) has a similar form as in Chiarella and Khomin (1996). In order to obtain the specific expression, we need to compute integrals of the form:

$$
I_{m}(t, T, \delta)=\int_{t-\delta}^{t} \frac{1}{C} \cdot \frac{1}{m !}\left(\frac{m}{T}\right)^{m+1}(t-s)^{m} e^{-\frac{m(t-s)}{T}} e^{\lambda s} d s
$$

if $m \geq 1$, and

$$
I_{0}(t, T, \delta)=\int_{t-\delta}^{t} \frac{1}{C} \cdot \frac{1}{T} e^{-\frac{t-s}{T}} e^{\lambda s} d s
$$

if $m=0$, where $C$ is given earlier as the normalising constant $C(m, T, \delta)$. From equation (23) we have (with new variable $u=t-s$ )

$$
\begin{aligned}
I_{0}(t, T, \delta) & =\int_{0}^{\delta} \frac{1}{C} \cdot \frac{1}{T} e^{-\frac{u}{T}} e^{\lambda(t-u)} d u \\
= & \frac{1}{C T} e^{\lambda t} \int_{0}^{\delta} e^{-u\left(\lambda+\frac{1}{T}\right)} d u=\frac{e^{\lambda t}}{C T}\left[\frac{e^{-u\left(\lambda+\frac{1}{T}\right)}}{-\left(\lambda+\frac{1}{T}\right)}\right]_{0}^{\delta} \\
= & \frac{e^{\lambda t}}{C T\left(\lambda+\frac{1}{T}\right)}\left(1-e^{-\delta\left(\lambda+\frac{1}{T}\right)}\right)=\frac{C(0, T, \delta(1+\lambda T))}{C(0, T, \delta)}(1+\lambda T)^{-1} e^{\lambda t} .
\end{aligned}
$$

If $m \geq 1$, then by introducing the new variable $u=t-s$ again,

$$
\begin{aligned}
I_{m}(t, T, \delta) & =\int_{0}^{\delta} \frac{1}{C} \frac{1}{m !}\left(\frac{m}{T}\right)^{m+1} u^{m} e^{-\frac{m u}{T}} e^{\lambda(t-u)} d u \\
= & \int_{0}^{\delta} \frac{1}{C} \frac{1}{m !}\left(\frac{m}{T}\right)^{m+1} u^{m} e^{-\frac{m u}{T}\left(1+\frac{\lambda T}{m}\right)} d u \cdot e^{\lambda t} .
\end{aligned}
$$

Introduce next the new variable $v=u \cdot\left(1+\frac{\lambda T}{m}\right)$ to obtain

$$
\begin{aligned}
I_{m}(t, T, \delta) & =\int_{0}^{\delta\left(1+\frac{\lambda T}{m}\right)} \frac{1}{C} \frac{1}{m !}\left(\frac{m}{T}\right)^{m+1} \frac{v^{m}}{\left(1+\frac{\lambda T}{m}\right)^{m}} e^{-\frac{m v}{T}} \frac{d v}{\left(1+\frac{\lambda T}{m}\right)} \cdot e^{\lambda t} \\
= & \frac{C\left(m, T, \delta\left(1+\frac{\lambda T}{m}\right)\right)}{C(m, T, \delta)} \cdot \frac{1}{\left(1+\frac{\lambda T}{m}\right)^{m+1}} e^{\lambda t} .
\end{aligned}
$$

Introduce the following notation: 


$$
A_{i}(\lambda)=\left\{\begin{array}{l}
\lambda+k_{i} \frac{C\left(l_{i}, S_{i}, \Delta_{i}\left(1+\frac{\lambda S_{i}}{l_{i}}\right)\right)}{C\left(l_{i}, S_{i}, \Delta_{i}\right)}\left(1+\frac{\lambda S_{i}}{l_{i}}\right)^{-l_{i}-1} \quad \text { if } l_{i} \geq 1 \\
\lambda+k_{i} \frac{C\left(0, S_{i}, \Delta_{i}\left(1+\lambda S_{i}\right)\right)}{C\left(0, S_{i}, \Delta_{i}\right)}\left(1+\lambda S_{i}\right)^{-1} \quad \text { if } l_{i}=0
\end{array}\right.
$$

and

$$
B_{i}(\lambda)=\left\{\begin{array}{l}
-k_{i} \gamma_{i} \frac{\left.C\left(m_{i}, T_{i}, \delta_{i}\left(1+\frac{\lambda T_{i}}{m_{i}}\right)\right)\left(1+\frac{\lambda T_{i}}{m_{i}}\right)^{-m_{i}-1}, \delta_{i}\right)}{\text { if } m_{i} \geq 0} \\
-k_{i} \gamma_{i} \frac{C\left(0, T_{i}, \delta_{i}\left(1+\lambda T_{i}\right)\right)}{C\left(0, T_{i}, \delta_{i}\right)}\left(1+\lambda T_{i}\right)^{-1} \quad \text { if } m_{i}=0 .
\end{array}\right.
$$

Then the eigenvalue equation of the linearized differential-difference equation system (21) has the form:

$$
A_{i}(\lambda) v_{i}+B_{i}(\lambda) \sum_{j \neq i} v_{j}=0 \quad(i=1,2, \ldots, n)
$$

which is equivalent to the determinantal equation

$$
\operatorname{det}\left(\begin{array}{cccc}
A_{1}(\lambda) & B_{1}(\lambda) & \ldots & B_{1}(\lambda) \\
B_{2}(\lambda) & A_{2}(\lambda) & \ldots & B_{2}(\lambda) \\
\vdots & \vdots & \ddots & \vdots \\
B_{n}(\lambda) & B_{n}(\lambda) & \ldots & A_{n}(\lambda)
\end{array}\right)=0 .
$$

Notice that this determinant has the same structure as in the case of infinitely continuously distributed lags (see Chiarella and Szidarovszky, 2000) ${ }^{4}$, so equation (28) can be rewritten as equation (22).

The main local stability result may now be stated as:

Corollary. The equilibrium of the system (3) is locally asymptotically stable if all roots of equation (22) have negative real parts.

Notice that equation (22) can be solved by solving $n+1$ equations of more simple structure:

$$
A_{i}(\lambda)-B_{j}(\lambda)=0 \quad(j=1,2, \ldots, n)
$$

4 Indeed the expressions for $A_{i}(\lambda), B_{i}(\lambda)$ reduce to the corresponding ones in Chiarella and Szidarovszky (2001) when $\delta_{i} \rightarrow \infty$ and $\Delta_{i} \rightarrow \infty$. 
and

$$
1+\sum_{j=1}^{n} \frac{B_{j}(\lambda)}{A_{j}(\lambda)-B_{j}(\lambda)}=0
$$

We will now consider the symmetric case when $m_{i} \equiv m$, $l_{i} \equiv l, k_{i} \equiv k, \gamma_{i} \equiv \gamma, S_{i} \equiv S, T_{i} \equiv T, \delta_{i} \equiv \delta$, and $\Delta_{i} \equiv \Delta$. Let

$$
q=\left\{\begin{array}{ll}
l & \text { if } l \geq 1 \\
1 & \text { if } l=0
\end{array} \quad \text { and } \quad r= \begin{cases}m & \text { if } m \geq 1 \\
1 & \text { if } m=0 .\end{cases}\right.
$$

With symmetric initial conditions system (21) becomes one-dimensional and similarly to equation (22) we can prove that in this case equation (28) has the special form

$$
\begin{aligned}
& \lambda\left(1+\frac{\lambda S}{q}\right)^{l+1}\left(1+\frac{\lambda T}{r}\right)^{m+1}+k \frac{C\left(l, S, \Delta\left(1+\frac{\lambda S}{q}\right)\right)}{C(l, S, \Delta)}\left(1+\frac{\lambda T}{r}\right)^{m+1} \\
& -k \gamma(n-1) \frac{C\left(m, T, \delta\left(1+\frac{\lambda T}{r}\right)\right)}{C(m, T, \delta)}\left(1+\frac{\lambda S}{q}\right)^{l+1}=0 .
\end{aligned}
$$

Thus we have the following result:

Theorem 2. The equilibrium of the symmetric dynamic oligopoly with bounded continuously distributed lags is locally asymptotically stable if the roots of the polynomial-exponential equation (31) have negative real parts.

If both lags $\delta$ and $\Delta$ are allowed to tend to infinity then the polynomial-exponential equation (31) reduces to the polynomial equation for the corresponding infinitely continuously distributed lag case in Chiarella and Szidarovszky (2001).

\section{The Birth of Limit Cycles}

In general equation (31) will have an infinite number of roots, many of which are possibly complex. Thus this class of dynamic oligopolies is quite prone to exhibiting fluctuating output. In this situation it becomes relevant to consider whether such 
fluctuations, could turn into limit cycle motion as certain parameters vary. The aim of this section is to consider this question.

For the sake of simplicity we restrict our analysis to the symmetric case. It is well-known from bifurcation theory (see, for example Hale and Verduyn Lunel, 1993) that limit cycles are possible if there are non-zero pure complex eigenvalues with additional conditions that will be presented later. Assume therefore that $\lambda=i \alpha$ with some real nonzero $\alpha$. In order to investigate equation (31) under this situation introduce the following notation:

$$
\left(1+\frac{i \alpha S}{q}\right)^{l+1}=A^{(r)}(\alpha)+i A^{(i)}(\alpha)
$$

with

$$
A^{(r)}(\alpha)=\sum_{v=0}^{\left[\frac{l+1}{2}\right]}\left(\begin{array}{c}
l+1 \\
2 v
\end{array}\right) \frac{\alpha^{2 v} S^{2 v}}{q^{2 v}}(-1)^{v}
$$

and

$$
A^{(i)}(\alpha)=\sum_{v=0}^{\left[\frac{l}{2}\right]}\left(\begin{array}{c}
l+1 \\
2 v+1
\end{array}\right) \frac{\alpha^{2 v+1} S^{2 v+1}}{q^{2 v+1}}(-1)^{v} .
$$

Similarly let

$$
\left(1+\frac{i \alpha T}{r}\right)^{m+1}=B^{(r)}(\alpha)+i B^{(i)}(\alpha)
$$

with

$$
B^{(r)}(\alpha)=\sum_{v=0}^{\left[\frac{m+1}{2}\right]}\left(\begin{array}{c}
m+1 \\
2 v
\end{array}\right) \frac{\alpha^{2 v} T^{2 v}}{r^{2 v}}(-1)^{v}
$$

and

$$
B^{(i)}(\alpha)=\sum_{v=0}^{\left[\frac{m}{2}\right]}\left(\begin{array}{c}
m+1 \\
2 v+1
\end{array}\right) \frac{\alpha^{2 v+1} T^{2 v+1}}{r^{2 v+1}}(-1)^{v} .
$$

Notice furthermore that

$$
C\left(l, S, \Delta\left(1+\frac{i \alpha S}{q}\right)\right)=1-e^{-\frac{l \Delta\left(1+\frac{i \alpha S}{q}\right)}{S}} \sum_{k=0}^{l} \frac{1}{k !} \frac{l^{k} \Delta^{k}\left(1+\frac{i \alpha S}{q}\right)^{k}}{S^{k}} .
$$

Introduce next the following additional notation: 


$$
\begin{aligned}
& D_{1}^{(r)}(\alpha)=\sum_{k=0}^{l}\left(\frac{1}{k !} \frac{l^{k} \Delta^{k}}{S^{k}} \sum_{v=0}^{\left[\frac{k}{2}\right]}\left(\begin{array}{c}
k \\
2 v
\end{array}\right) \frac{\alpha^{2 v} S^{2 v}}{q^{2 v}}(-1)^{v}\right), \\
& D_{1}^{(i)}(\alpha)=\sum_{k=0}^{l}\left(\frac{1}{k !} \frac{l^{k} \Delta^{k}}{S^{k}} \sum_{v=0}^{\left.\frac{k-1}{2}\right]}\left(\begin{array}{c}
k \\
2 v+1
\end{array}\right) \frac{\alpha^{2 v+1} S^{2 v+1}}{q^{2 v+1}}(-1)^{v}\right),
\end{aligned}
$$

and notice that

$$
e^{\frac{-l \Delta\left(1+\frac{i \alpha S}{q}\right)}{S}}=e^{-\frac{l \Delta}{S}}\left(\cos \frac{\alpha l \Delta}{q}-i \sin \frac{\alpha l \Delta}{q}\right)
$$

Similarly,

$$
C\left(m, T, \delta\left(1+\frac{i \alpha T}{r}\right)\right)=1-e^{-\frac{m \delta\left(1+\frac{i \alpha T}{r}\right)}{T}} \sum_{k=0}^{m} \frac{1}{k !} \frac{m^{k} \delta^{k}\left(1+\frac{i \alpha T}{r}\right)^{k}}{T^{k}} .
$$

Introduce the further notation,

$$
\begin{aligned}
& D_{2}^{(r)}(\alpha)=\sum_{k=0}^{m}\left(\frac{1}{k !} \frac{m^{k} \delta^{k}}{T^{k}} \sum_{v=0}^{\left.\frac{k}{2}\right]}\left(\begin{array}{c}
k \\
2 v
\end{array}\right) \frac{\alpha^{2 v} T^{2 v}}{r^{2 v}}(-1)^{v}\right), \\
& D_{2}^{(i)}(\alpha)=\sum_{k=0}^{m}\left(\frac{1}{k !} \frac{m^{k} \delta^{k}}{T^{k}} \sum_{v=0}^{\left.\frac{k-1}{2}\right]}\left(\begin{array}{c}
k \\
2 v+1
\end{array}\right) \frac{\alpha^{2 v+1} T^{2 v+1}}{r^{2 v+1}}(-1)^{v}\right),
\end{aligned}
$$

and notice that

$$
e^{\frac{-m \delta\left(1+\frac{i \alpha T}{r}\right)}{T}}=e^{-\frac{m \delta}{T}}\left(\cos \frac{\alpha m \delta}{r}-i \sin \frac{\alpha m \delta}{r}\right) .
$$

Using the above notation, equation (31) with $\lambda=i \alpha$ can be rewritten as

$$
\begin{aligned}
& i \alpha\left(A^{(r)}(\alpha)+i A^{(i)}(\alpha)\right)\left(B^{(r)}(\alpha)+i B^{(i)}(\alpha)\right)+ \\
& \frac{k}{C(l, S, \Delta)}\left[1-e^{-\frac{l \Delta}{S}}\left(\cos \frac{\alpha l \Delta}{q}-i \sin \frac{\alpha l \Delta}{q}\right)\left(D_{1}^{(r)}(\alpha)+i D_{1}^{(i)}(\alpha)\right)\right]\left(B^{(r)}(\alpha)+i B^{(i)}(\alpha)\right) \\
& -\frac{k \gamma(n-1)}{C(m, T, \delta)}\left[1-e^{-\frac{m \delta}{T}}\left(\cos \frac{\alpha m \delta}{r}-i \sin \frac{\alpha m \delta}{r}\right)\left(D_{2}^{(r)}(\alpha)+i D_{2}^{(i)}(\alpha)\right)\right]\left(A^{(r)}(\alpha)+i A^{(i)}(\alpha)\right)=0 .
\end{aligned}
$$

Equating the real and imaginary parts to zero we have the following equations:

$$
k \gamma(n-1)=\frac{E_{1}(\alpha)}{E_{2}(\alpha)}=\frac{F_{1}(\alpha)}{F_{2}(\alpha)}
$$

with 


$$
\begin{aligned}
E_{1}(\alpha)= & -\alpha\left(A^{(r)}(\alpha) B^{(i)}(\alpha)+A^{(i)}(\alpha) B^{(r)}(\alpha)\right)+\frac{k}{C(l, S, \Delta)}\left\{B^{(r)}(\alpha)\right. \\
& -e^{-\frac{l \Delta}{S}}\left[\cos \frac{\alpha l \Delta}{q}\left(D_{1}^{(r)}(\alpha) B^{(r)}(\alpha)-D_{1}^{(i)}(\alpha) B^{(i)}(\alpha)\right)\right. \\
& \left.\left.+\sin \frac{\alpha l \Delta}{q}\left(D_{1}^{(r)}(\alpha) B^{(i)}(\alpha)-D_{1}^{(i)}(\alpha) B^{(r)}(\alpha)\right)\right]\right\} \\
\left.E_{2}(\alpha)=\frac{1}{C(m,}, T, \delta\right) & \left\{A^{(r)}(\alpha)-e^{-\frac{m \delta}{T}}\left[\cos \frac{\alpha m \delta}{r}\left(D_{2}^{(r)}(\alpha) A^{(r)}(\alpha)-D_{2}^{(i)}(\alpha) A^{(i)}(\alpha)\right)\right.\right. \\
& \left.\left.+\sin \frac{\alpha m \delta}{r}\left(D_{2}^{(r)}(\alpha) A^{(i)}(\alpha)+D_{2}^{(i)}(\alpha) A^{(r)}(\alpha)\right)\right]\right\}, \\
F_{1}(\alpha)= & \alpha\left(A^{(r)}(\alpha) B^{(r)}(\alpha)-A^{(i)}(\alpha) B^{(i)}(\alpha)\right)+\frac{k}{C(l, S, \Delta)}\left\{B^{(i)}(\alpha)\right. \\
& -e^{-\frac{l \Delta}{S}}\left[\cos \frac{\alpha l \Delta}{q}\left(D_{1}^{(r)}(\alpha) B^{(i)}(\alpha)+D_{1}^{(i)}(\alpha) B^{(r)}(\alpha)\right)\right. \\
& \left.\left.-\sin \frac{\alpha l \Delta}{q}\left(D_{1}^{(r)}(\alpha) B^{(r)}(\alpha)-D_{1}^{(i)}(\alpha) B^{(i)}(\alpha)\right)\right]\right\}
\end{aligned}
$$

and

$$
\begin{gathered}
F_{2}(\alpha)=\frac{1}{C(m, T, \delta)}\left\{A^{(i)}(\alpha)-e^{-\frac{m \delta}{T}}\left[\cos \frac{\alpha m \delta}{r}\left(D_{2}^{(r)}(\alpha) A^{(i)}(\alpha)+D_{2}^{(i)}(\alpha) A^{(r)}(\alpha)\right)\right.\right. \\
\left.\left.-\sin \frac{\alpha m \delta}{r}\left(D_{2}^{(r)}(\alpha) A^{(r)}(\alpha)-D_{2}^{(i)}(\alpha) A^{(i)}(\alpha)\right)\right]\right\}
\end{gathered}
$$

Any real root of equation (32) must satisfy

$$
E_{1}(\alpha) F_{2}(\alpha)-E_{2}(\alpha) F_{1}(\alpha)=0
$$

which is a mixed polynomial-trigonometric equation for $\alpha$. Notice that equation (33) might have other roots which are not solutions of equation (32). These additional roots can be identified by simple substitution. Let $\alpha^{*}$ be a root of equation (32), then $\lambda^{*}=i \alpha^{*}$ is an eigenvalue, and there is a functional relation between $\alpha^{*}$ and the critical bifurcation value of the parameter $\gamma$.

Using conditions stated in Hale and Verduyn Lunel (1993) ${ }^{5}$, we may assert that limit cycles are born if equation (32) has no other real root $\alpha=\mu \alpha^{*}$ with any integer $\mu$ for $\gamma=\gamma^{*}$, and if the real part of the derivative $d \lambda / d \gamma$ is non-zero at the root $\lambda^{*}$. The 
condition on the other eigenvalues is hard to verify analytically, but computer methods can be used in particular cases.

Notice that

$$
\frac{d \lambda}{d \gamma}=\frac{P_{1}(\lambda)}{P_{2}(\lambda)}
$$

where

$$
P_{1}(\lambda)=k(n-1) \frac{C\left(m, T, \delta\left(1+\frac{\lambda T}{r}\right)\right)}{C(m, T, \delta)}\left(1+\frac{\lambda S}{q}\right)^{l+1}
$$

and

$$
\begin{aligned}
& P_{2}(\lambda)= \\
& \left(1+\frac{\lambda S}{q}\right)^{l+1}\left(1+\frac{\lambda T}{r}\right)^{m+1}+\lambda(l+1) \frac{S}{q}\left(1+\frac{\lambda S}{q}\right)^{l}\left(1+\frac{\lambda T}{r}\right)^{m+1}+\lambda(m+1) \frac{T}{r}\left(1+\frac{\lambda S}{q}\right)^{l+1}\left(1+\frac{\lambda T}{r}\right)^{m} \\
& +\frac{k}{C(l, S, \Delta)}\left[\frac{d}{d \lambda} C\left(l, S, \Delta\left(1+\frac{\lambda S}{q}\right)\right)\left(1+\frac{\lambda T}{r}\right)^{m+1}+C\left(l, S, \Delta\left(1+\frac{\lambda S}{q}\right)\right)(m+1) \frac{T}{r}\left(1+\frac{\lambda T}{r}\right)^{m}\right] \\
& -\frac{k \gamma(n-1)}{C(m, T, \delta)}\left[\frac{d}{d \lambda} C\left(m, T, \delta\left(1+\frac{\lambda T}{r}\right)\right)\left(1+\frac{\lambda S}{q}\right)^{l+1}+C\left(m, T, \delta\left(1+\frac{\lambda T}{r}\right)\right)(l+1) \frac{S}{q}\left(1+\frac{\lambda S}{q}\right)^{l}\right] .
\end{aligned}
$$

Hence we may appeal to the Hopf bifurcation theorem (Hale and Verduyn Lunel, 1993) to state the following result:

Theorem 3. Let $\alpha^{*}$ be a real root of equation (32) with $\gamma^{*}$ being the derivative of the best response function at the equilibrium, assume there is no other root $\alpha=\mu \alpha^{*}$ for any integer $\mu$, furthermore at $\gamma=\gamma^{*}$,

$$
\operatorname{Re} \frac{P_{1}\left(i \alpha^{*}\right)}{P_{2}\left(i \alpha^{*}\right)} \neq 0 \text {. }
$$

Then there is a limit cycle around the equilibrium.

The Hopf bifurcation theorem does not enable us to say anything about the stability of the limit cycle. To do so in the context of these dynamic oligopoly models would require

\footnotetext{
${ }^{5}$ See section 11.1.
} 
specification of the best response function and consideration of conditions arising from normal form theory. However in the present context this may be difficult and numerical simulations are probably the only practical approach. We consider one specific example in Section 8.

\section{6. $\quad$ Pure Complex Roots in a Special Case}

In this section we consider the special case of $S=0$, when no time-lag is assumed in obtaining and implementing information on the firms' own output. In this case equation (31) simplifies to:

$$
(\lambda+k)\left(1+\frac{\lambda T}{r}\right)^{m+1}=k \gamma(n-1) \frac{1-e^{-\frac{m \delta\left(1+\frac{\lambda T}{r}\right)}{T}} \sum_{k=0}^{m} \frac{m^{k} \delta^{k}}{k ! T^{k}}\left(1+\frac{\lambda T}{r}\right)^{k}}{1-e^{-\frac{m \delta}{T}} \sum_{k=0}^{m} \frac{m^{k} \delta^{k}}{k ! T^{k}}} .
$$

Consider first the case of $\boldsymbol{m}=\mathbf{0}$. Then the above equation is quadratic:

$$
(\lambda+k)(1+\lambda T)=k \gamma(n-1) .
$$

If $\lambda=i \alpha$, then this equation becomes

$$
(i \alpha+k)(1+i \alpha T)=k \gamma(n-1) .
$$

Equating the real and imaginary parts we have

$$
k-\alpha^{2} T=k \gamma(n-1) \quad \text { and } \quad \alpha(1+k T)=0 .
$$

Therefore $\alpha^{*}=0$ and $\gamma^{*}=\frac{1}{n-1}$ is the only solution, which implies that the birth of limit cycles is not guaranteed in this case given that $\gamma<0$, and the pure complex root is zero.

Consider next the case of $m=1$. Then equation (35) reduces to

$$
(\lambda+k)(1+\lambda T)^{2}=k \gamma(n-1) \frac{1-e^{-\frac{\delta(1+\lambda T)}{T}}\left(1+\frac{\delta}{T}(1+\lambda T)\right)}{1-e^{-\frac{\delta}{T}}\left(1+\frac{\delta}{T}\right)} .
$$

If $\lambda=i \alpha$, then the left hand side of (36) is

$$
(i \alpha+k)\left(1+2 i \alpha T-\alpha^{2} T^{2}\right)=\left(-\alpha^{2} 2 T+k-k \alpha^{2} T^{2}\right)+i\left(\alpha-\alpha^{3} T^{2}+2 k \alpha T\right) .
$$

Similarly the right hand side is 


$$
\begin{aligned}
& \frac{k \gamma(n-1)}{1-e^{-\frac{\delta}{T}}\left(1+\frac{\delta}{T}\right)}\left[1-e^{-\frac{\delta}{T}}(\cos \delta \alpha-i \sin \delta \alpha)\left(1+\frac{\delta}{T}+\delta \alpha i\right)\right] \\
& =\frac{k \gamma(n-1)}{1-e^{-\frac{\delta}{T}}\left(1+\frac{\delta}{T}\right)}\left\{1-e^{-\frac{\delta}{T}}\left[\cos \delta \alpha\left(1+\frac{\delta}{T}\right)+\sin \delta \alpha(\delta \alpha)\right]+i e^{-\frac{\delta}{T}}\left[-\cos \delta \alpha(\delta \alpha)+\sin \delta \alpha\left(1+\frac{\delta}{T}\right)\right]\right\} .
\end{aligned}
$$

Comparing the real and imaginary parts we obtain

$$
\begin{aligned}
k \gamma(n-1) & =\frac{\left(-\alpha^{2} 2 T+k-k \alpha^{2} T^{2}\right)\left(1-e^{-\frac{\delta}{T}}\left(1+\frac{\delta}{T}\right)\right)}{1-e^{-\frac{\delta}{T}}\left[\cos \delta \alpha\left(1+\frac{\delta}{T}\right)+\sin \delta \alpha(\delta \alpha)\right]} \\
= & \frac{\left(\alpha-\alpha^{3} T^{2}+2 k \alpha T\right)\left(1-e^{-\frac{\delta}{T}}\left(1+\frac{\delta}{T}\right)\right)}{e^{-\frac{\delta}{T}}\left[-\cos \delta \alpha(\delta \alpha)+\sin \delta \alpha\left(1+\frac{\delta}{T}\right)\right]}
\end{aligned}
$$

This equation can be rewritten as a single polynomial-trigonometric equation for $\alpha$, viz.,

$$
\begin{aligned}
& G(\alpha) \equiv\left[k-\alpha^{2}\left(2 T+k T^{2}\right)\right] e^{-\frac{\delta}{T}}\left[-\delta \alpha \cos \delta \alpha+\left(1+\frac{\delta}{T}\right) \sin \delta \alpha\right] \\
& -\left[\alpha(1+2 k T)-\alpha^{3} T^{2}\right]\left\{1-e^{-\frac{\delta}{T}}\left[\left(1+\frac{\delta}{T}\right) \cos \delta \alpha+\delta \alpha \sin \delta \alpha\right]\right\}=0 .
\end{aligned}
$$

All eigenvalues satisfy equation (38). However there might be additional roots of (38) which are not eigenvalues. They can be identified by simple substitution into equation (36). For instance, $\alpha=0$ is always a root, but $\lambda=0$ solves equation (36) for only $\gamma=1 /(n-1)$ which is not possible, since $\gamma<0$.

We need at least one non-zero real root in order to establish the birth of limit cycles. Let $\alpha^{*}$ be a non-zero real root, then the corresponding value $\gamma^{*}$ of the bifurcation parameter is obtained from equation (37). The derivative $d \lambda / d \gamma$ (evaluated at the equilibrium) can also be obtained from equation (36) as

$$
\frac{d \lambda}{d \gamma}=\frac{P_{1}(\lambda)}{P_{2}(\lambda)}
$$

with

$$
P_{1}(\lambda)=\left[1-e^{-\frac{\delta(1+\lambda T)}{T}}\left(1+\frac{\delta}{T}(1+\lambda T)\right] k(n-1)\right.
$$

and 


$$
P_{2}(\lambda)=\left[1-e^{-\frac{\delta}{T}}\left(1+\frac{\delta}{T}\right)\right]\left[(1+\lambda T)^{2}+(\lambda+k) 2 T(1+\lambda T)\right]-k \gamma(n-1) \cdot \frac{\delta^{2}}{T} e^{-\frac{\delta(1+\lambda T)}{T}}(1+\lambda T) .
$$

If equation (36) has no other root $\alpha=\mu \alpha^{*}$ with any integer $\mu$ for $\gamma=\gamma^{*}$, and if the real part of this derivative at $\alpha=\alpha^{*}$ and $\gamma=\gamma^{*}$ is non-zero, then there are limit cycles around the equilibrium.

Note that by letting $\delta \rightarrow \infty$ in equation (38) we obtain

$$
\alpha^{* 2}=\frac{2 k T+1}{T^{2}}
$$

and then from the first equality in (37)

$$
\gamma^{*}=-\frac{2}{(n-1)}\left[\frac{1}{k T}+k T+2\right]
$$

these being indeed the results obtained for this case by Chiarella and Szidarovszky (2001). In the bounded lag case equation (38) possibly has an infinite number of roots and hence, an infinite number of bifurcation values $\gamma^{*}$ are possible. In order to appreciate the much richer set of possibilities it is necessary to consider a numerical example.

\section{Numerical Example}

We consider from Section 6 the special case of $m=1$ with $T=1, k=3$ and $n=10$.

The key to understanding the overall structure of the eigenvalues and the critical values of $\gamma$ is to consider how the roots of equation (38) evolve as the parameter $\delta$ increases from 0 through 5 (the value that for our purposes may be regarded as infinity).

Since we are only concerned with non-zero roots the ensuing discussion ignores the root at $\alpha=0$. We note that equation (38) is symmetric with respect to $\alpha$, so we only consider positive values. Figure 3a shows a plot of $G(\alpha)$ at $\delta=2.5$ for $\alpha$ up to 30 illustrating the cyclically increasing nature of this function. Some elementary manipulations on $G(\alpha)$ for large $\alpha$ indicate easily that $G(\alpha)$ must have an infinite number of real roots. A root $\alpha \neq 0$ may lead to the birth of limit cycles if none of the other roots is integer multiple of $\alpha^{*}$ and gives the same critical value of $\gamma$. 


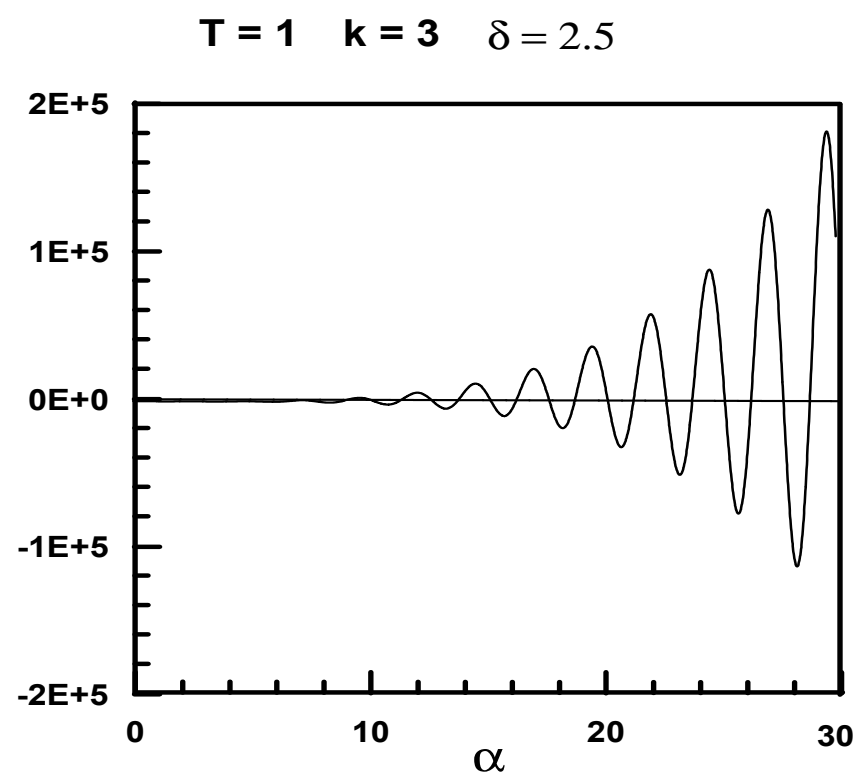

Figure 3a

Figure $3 \mathrm{~b}$ shows the more detailed structure of $G(\alpha)$ for $\alpha$ up to about 7.5, for three separate values of $\delta$. At $\delta=3.0$ (the blue curve) we can view the intersections with the horizontal axis resulting in the first seven roots. At $\delta=3.17$ (the red curve) we see how the first two roots disappear via a tangency, the third root declines, roots four and five decline and move closer together, and roots six and seven appear. At $\delta=3.40$ (the black curve) the third root continuous to decline, the fourth and fifth roots are close to disappearing and the sixth and seventh roots are just disappearing. For this set of parameters this is the only "reversal" in the order in which roots disappear that we have observed.

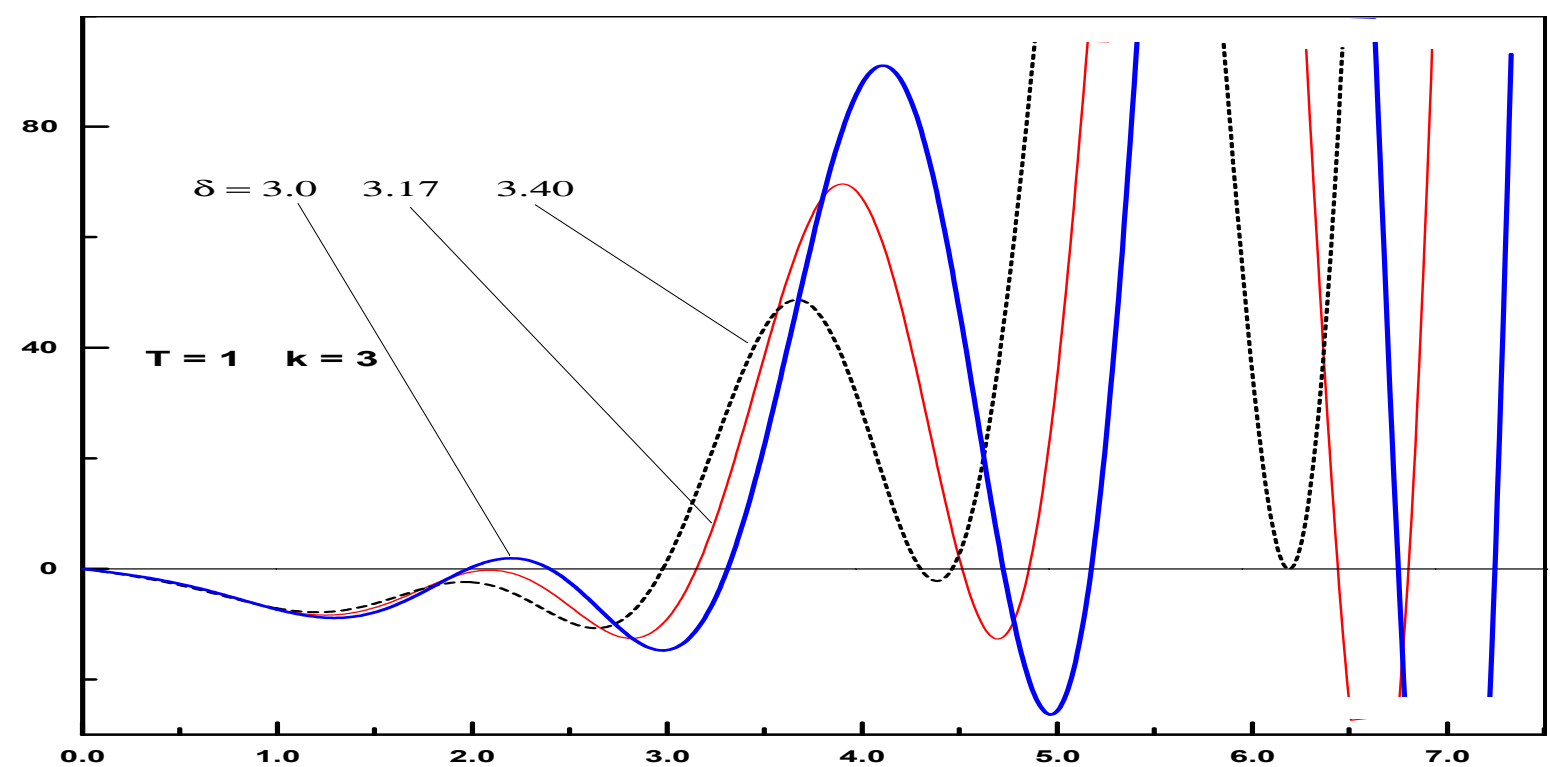

Figure 3b 
The foregoing observations on Figure 3b should then make fairly transparent how Figure $4 \mathrm{a}$ is constructed. The blue "tongue" shows the first (lower) and second (upper) roots which come together and then disappear at $\delta=3.17$. The black curve show the third root declining continuously to its limiting value at $\delta=5$, this limiting value is in fact close to $\sqrt{7}$ as would be expected from equation (40). The red tongue shows the fourth (lower) and fifth (upper) roots which came together and then disappear at $\delta=3.48$. The green tongue shows the sixth and seventh roots which come together and then disappear at $\delta=3.41$. The tongues for roots eight, nine and ten, eleven are also shown in Figure 4a. The full range of tongues up to $\alpha=25$ are shown in Figure $4 \mathrm{~b}$, this is close to the largest value of $\alpha$ that is relevant given that $\delta=5$ is considered to be infinity for the purposes of these illustrative calculations.

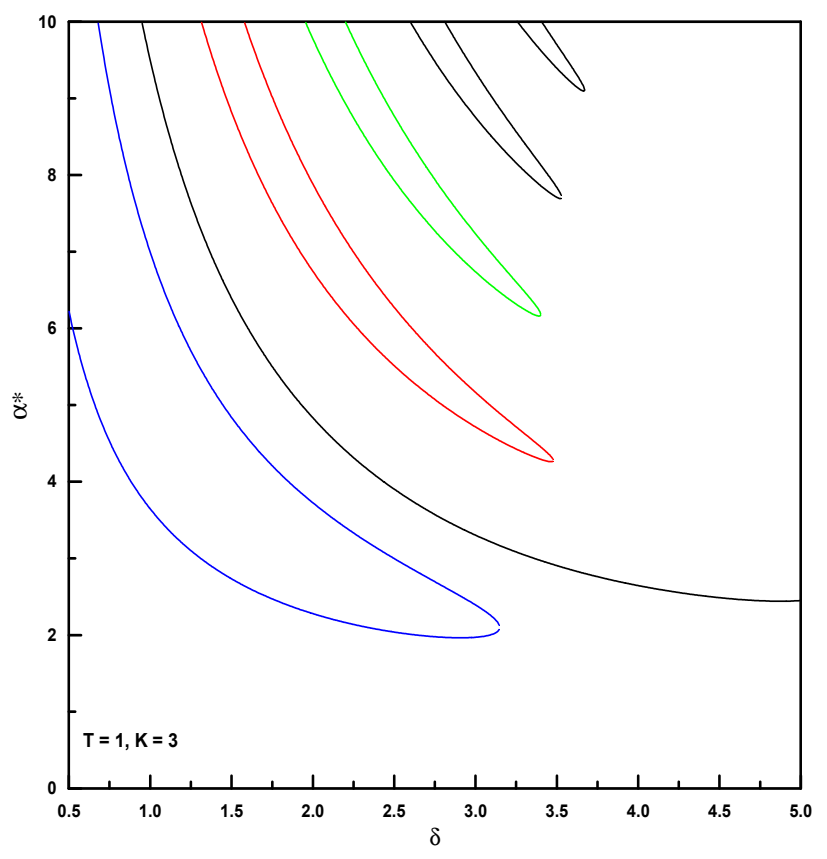

Figure 4a

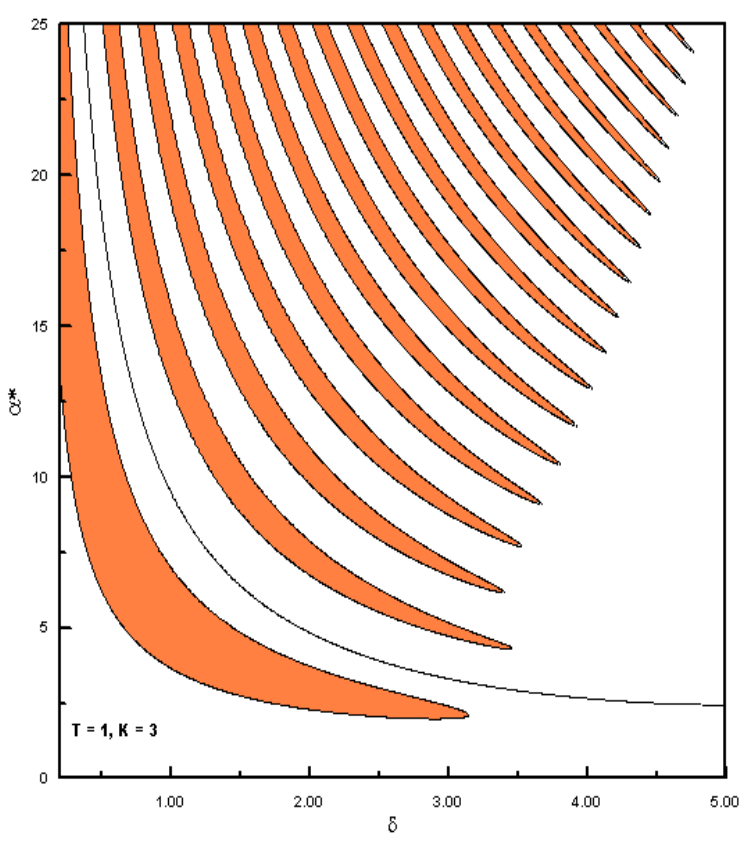

Figure 4b

We next use the values of $\alpha$ in Figure $4 \mathrm{a}$ to construct, via equation (37), the corresponding critical values of $\gamma$ at which a pair of pure complex roots occurs. These are shown in Figure 5a with the colours corresponding to the tongues (except in the case of root three) shown in Figure 4a. The limiting value to which the black curve 
(corresponding to the third root for $\alpha$ ) tends is in fact close to $-32 / 27$ as expected from equation (41). Figure $5 \mathrm{~b}$ shows the whole sequence of critical $\gamma$ curves corresponding to the sequence of tongues in Figure $4 \mathrm{~b}$.

In order to obtain a more detailed global picture of the dynamics it would be necessary to specify functional forms for the reaction function $g$ and calculate for example a bifurcation diagram with respect to $\delta$. This we do for one specific example in the next section.

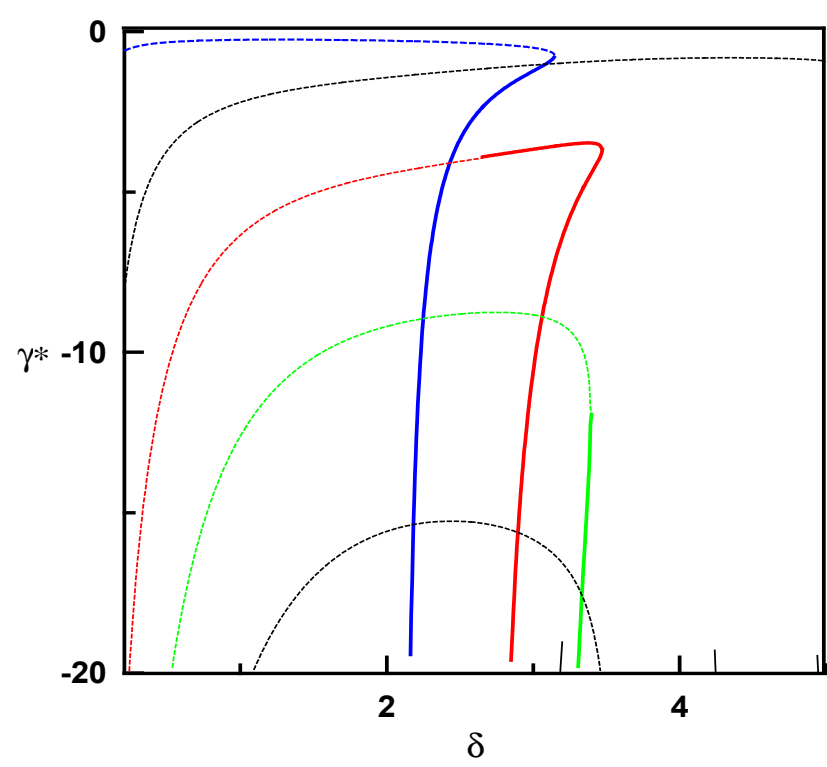

Figure 5a

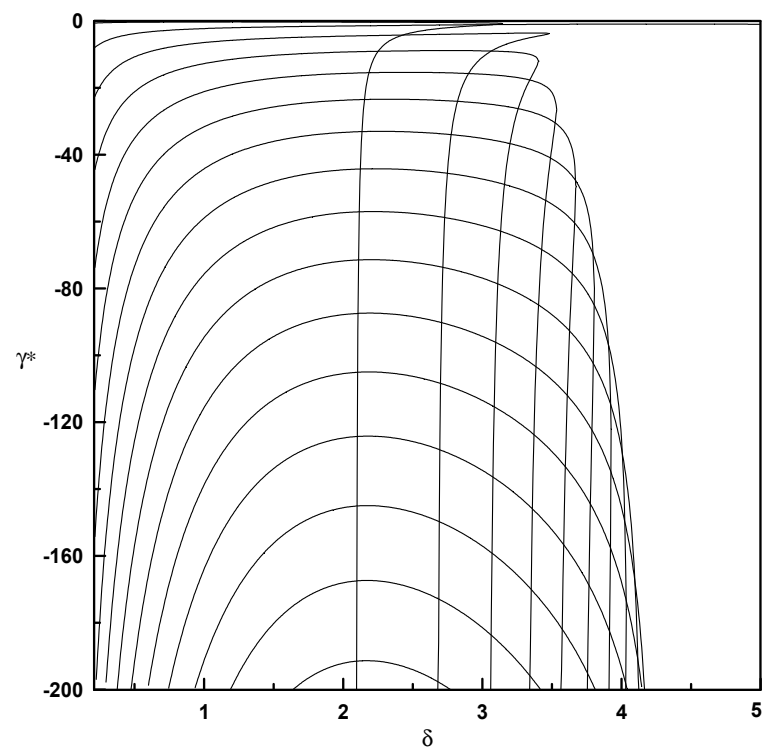

Figure 5b

\section{8. $\quad$ A Specific Reaction Function}

In order to generate a specific example we assume linear cost functions and the hyperbolic inverse demand function $f(Q)=\frac{A}{Q}$ with some $A>0$. The profit of firm $j$ is then given as

$$
\pi_{j}=x_{j} \frac{A}{x_{j}+Q_{j}}-c_{j} x_{j},
$$

(where $c_{j}$ is the marginal cost) and the best response of this firm is 


$$
g_{j}\left(Q_{j}\right)= \begin{cases}0, & \text { if } Q_{j} \geq A / c_{j}, \\ \sqrt{\frac{A Q_{j}}{c_{j}}}-Q_{j}, & \text { otherwise. }\end{cases}
$$

We continue to focus on the symmetric case considered in Section 6 where in addition we have set $c_{j}=c$. At a symmetric equilibrium $Q_{j}^{*}=(n-1) x^{*}$ and $Q^{*}=n x^{*}$, and $x^{*}$ is the solution of equation

$$
x=g((n-1) x),
$$

which by use of (42) yields

$$
x=\left(\sqrt{\frac{A(n-1) x}{c}}-(n-1) x\right),
$$

implying that

$$
x^{*}=\frac{A(n-1)}{c n^{2}} .
$$

It is easy to see that for $n>2$, the function $g_{j}$ is strictly decreasing in $Q_{j}$ in the neighborhood of the equilibrium, hence satisfying the condition on the best response function made in Section 3.

From equation (8) and (9) it is easy to verify that

$$
q_{i, m}^{(k)^{*}}=(n-1) x^{*}\left(1-e^{-\frac{m \delta}{T}} \sum_{j=1}^{k} \frac{\left(\frac{m \delta}{T}\right)^{j}}{j !}\right)
$$

for $k=0,1, \ldots, m$, so in the special case of $k=m$,

$$
q_{i, m}^{(m)^{*}}=(n-1) x^{*} C(m, T, \delta)
$$

implying that

$$
Q_{i}^{e^{*}}=(n-1) x^{*} .
$$

Using the reaction function (42) we have simulated the system of Lemma 2 for the case $S=0$ (as in Section 6) and $k=3, n=10$ and $m=1$ (as in Section 7) and $A / c=1$. The lag $\delta$ was taken as a bifurcation parameter and the bifurcation diagram in Figure 6 was calculated for $T=1$ and $T=1.5$ over the range $0<\delta \leq 5$. Recall that from a practical point 
of view $\delta=5$ may be regarded as the infinitely distributed lag case. We see that compared to this limiting case the bounded lag weighting schemes increase the likelihood of limit cycle motions, depending on the value of $T$. This one numerical example illustrates how the bounded lag weighting scheme can more readily yield fluctuating dynamics. We leave for future research a more extensive numerical study of this model, which should study other reaction functions and allow the firms to differ in various ways.

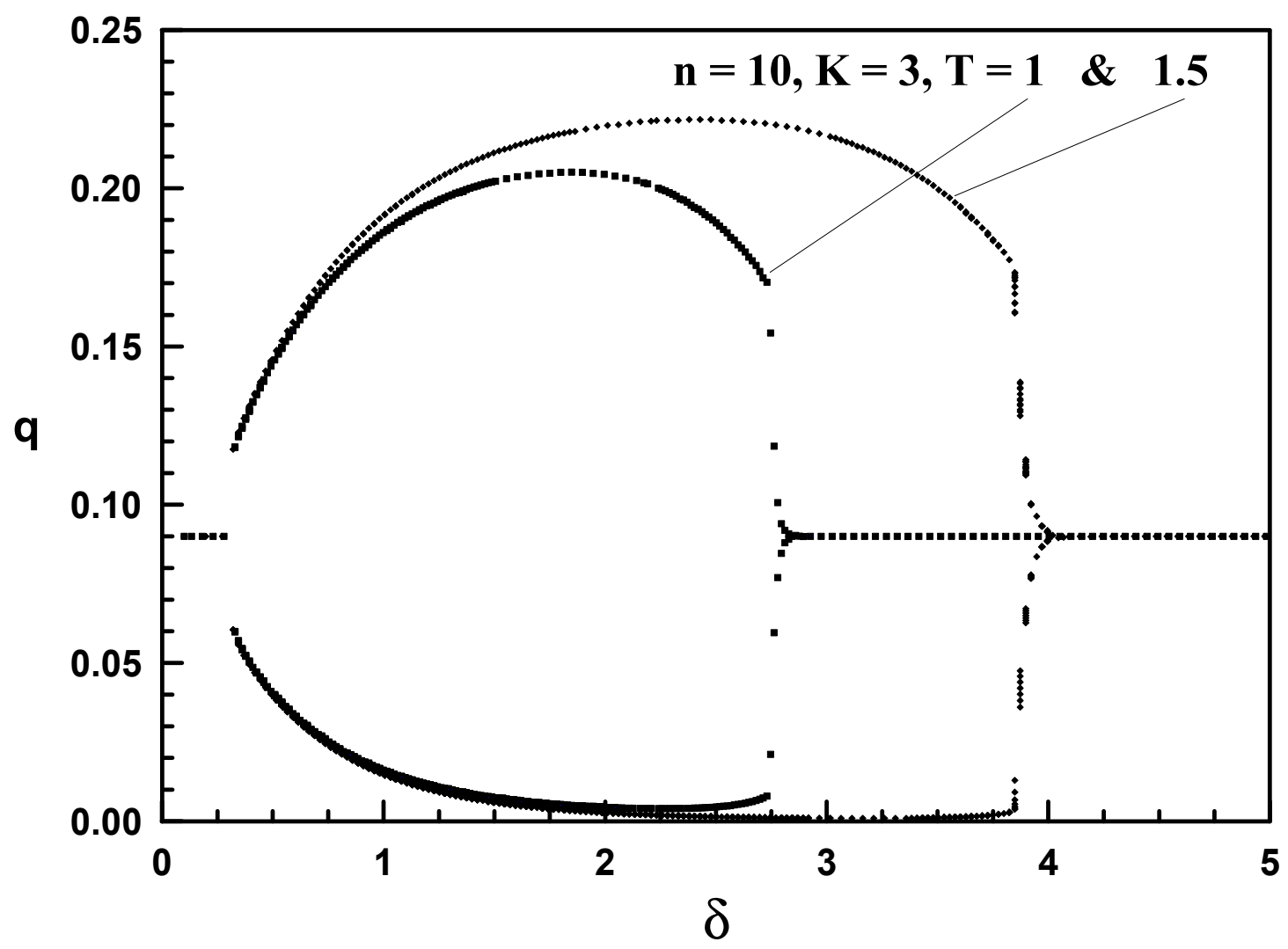

Figure 6

\section{Conclusions}

In this paper we have introduced a weighting function that caters for bounded continuously distributed lags in dynamic economic models. In this way past data are averaged only over a bounded interval thus avoiding the use of very old or less relevant economic data. We have used this weighting function to capture the effects of information and implementation lags about firms' own, as well as rivals', output in a 
fairly general class of dynamic oligopoly models. We have formulated the dynamics of the resulting oligopoly as a system of integro-differential equations and shown how this may be reduced to a system of ordinary differential-difference equations. We have then employed standard techniques from the theory of differential-difference equations to analyse the local stability of the equilibrium. We have then applied the Hopf-bifurcation theorem to determine conditions on the model parameters under which limit cycle motion may be born (or destroyed). We have considered in some detail the special case of a symmetric oligopoly and examined it numerically for a particular set of parameters. This example indicates how the dynamic structure becomes much more complex compared to the corresponding (and limiting) infinitely continuously distributed lag case. In that case there is just one critical value of which limit cycles are born (or destroyed), whereas in the bounded lag case there may be many such values. Dynamic oligopoly models with bounded continuously distributed lags are thus far more easily able to exhibit limit cycle and possibly more complex motion. We have given one simple numerical example that seems to confirm these insights from the theoretical discussion.

We believe we have taken an analytical investigation of the stability of this class of dynamic oligopoly models as far as is possible. It would be of interest to complement this study with a more extensive simulation study to see how Figures 3 to 6 are altered by allowing the firms to differ in some way, also by considering a higher value of the parameter $m$ in Section 6. It would also be of interest to analyse globally the types of attractors, as well as their basins of attraction, that may occur. Whilst in this paper we have only considered bounded continuously distributed lags within dynamic oligopoly models, we believe their implications within a range of other dynamic economic models should be examined. Such lags surely reflect more accurately the reality of economic decision making and it seems difficult to believe that the stability structure displayed by the example in Sections 7 and 8 is confined merely to dynamic oligopoly models. 


\section{0. $\underline{\text { References }}$}

Bellman, R. and K. L. Cooke (1963): Differential-Difference Equations. Academic Press, New York.

Chiarella, C. and A. Khomin (1996): An Analysis of the Complex Dynamic Behaviour of Nonlinear Oligopoly Models with Time Delays.Chaos, Solitons and Fractals, Vol. 7, No. 2, pp.2049-2065

Chiarella, C. and F. Szidarovszky (2001): Limit Cycles in Nonlinear Oligopolies with Continuously Distributed Time Delays. To appear in Modelling Uncertainty (M. Dror ed.), Kluwer Academic Publishers, Dordrecht.

Cushing, J. M. (1977): Integro-Differential Equations and Delay Models in Population Dynamics. Springer-Verlag, Berlin/ Heidelberg/ New York.

Hale, J. K. and S. M. Verduyn Lunel (1993): Introduction to Functional Differential Equations. Springer-Verlag, Berlin/Heidelberg/New York.

Invernizzi S.and A. Medio (1991): On Lags and Chaos in Economic Models. Journal of Mathematical Economics, Vol. 20, pp. 512-550.

Okuguchi, K. (1976): Expectations and Stability in Oligopoly Models. Springer-Verlag, Berlin /Heidelberg/New York.

Okuguchi, K. and F. Szidarovszky (1999): The Theory of Oligopoly with MultiProduct Firms ( $2^{\text {nd }}$ edition), Springer-Verlag, Berlin/ Heidelberg/ New York.

Puu, T. (2000): Attractors, Bifurcations and Chaos; Nonlinear Phenomena in Economics, Springer-Verlag, Berlin/ Heidelberg/ New York.

Russel, A. M., J. Rickard, and T. D. Howroyd (1986): The Effects of Delays on the Stability and Rate of Convergence to Equilibrium of Oligopolies. Economic Record, Vol. 62, pp. 194-198. 